\title{
Construction and properties of aconitase mutants of Escherichia coli
}

\author{
Megan J. Gruer, Alan J. Bradbury and John R. Guest \\ Author for correspondence: John R. Guest. Tel: +44 114222 4406/3. Fax: + 441142728697.
e-mail: J.R.Guest@Sheffield.ac.uk
}

The Krebs Institute for Biomolecular Research, Department of Molecular Biology and

Biotechnology, University of Sheffield, Western Bank, Sheffield S10 2TN, UK

\begin{abstract}
Escherichia coli contains two genes (acnA and acnB) encoding aconitase activities. An acnB mutant was engineered by replacing the chromosomal acnB gene by an internally deleted derivative containing a tet ${ }^{R}$ cassette. An acnAB double mutant was then made by transducing a previously constructed acn $A:: k^{R}{ }^{R}$ mutation into the acnB:: tet $^{R}$ strain. Western blotting confirmed that the ACnA and AcnB proteins were no longer produced by the corresponding mutants and PCR analysis showed that the chromosomal acnB gene had been replaced by the disrupted gene. Aerobic and anaerobic growth in glucose minimal medium were impaired but not abolished by the acn $B$ mutation, indicating that the lesion is partially complemented by the acnA ${ }^{+}$ gene, and growth was enhanced by glutamate. The acnAB double mutant would not grow on unsupplemented glucose minimal medium and although it responded to glutamate like a typical auxotroph under anaerobic conditions, under aerobic conditions no response to glutamate was observed before it was over-grown by 'revertants' lacking citrate synthase (acnAB g/tA). The acnAB double mutant retained a low but significant aconitase activity ( $\leqslant 5 \%$ of wildtype), designated AcnC. Enzymological and regulatory studies with acn-lacz fusions indicated that AcnB is the major aconitase, which is synthesized earlier in the growth cycle than Acn $A$, and subject to catabolite and anaerobic repression.
\end{abstract}

Keywords: aconitase, citrate synthase, citric acid cycle, glutamate auxotrophy, Escherichia coli

\section{INTRODUCTION}

Aconitases (EC 4.2.1.3) are widely distributed monomeric enzymes containing a single $[4 \mathrm{Fe}-4 \mathrm{~S}]$ centre and catalysing the reversible isomerization of citrate and isocitrate via cis-aconitate. They function in the citric acid and glyoxylate cycles. Escherichia coli has recently been shown to contain at least two genetically distinct aconitases, AcnA and AcnB (Gruer \& Guest, 1994). $\operatorname{Acn} A\left(M_{\mathrm{r}} 97500\right)$ is encoded by the acn $A$ gene, located at $28 \min (1350 \mathrm{~kb})$, whereas $\mathrm{AcnB}$ (predicted $M_{\mathrm{r}} 93500$, but $93000-105000$ by different methods) is the product of the $a c n B$ gene located at $2.85 \mathrm{~min}(131.6 \mathrm{~kb})$ in the $E$. coli genome (Prodromou et al., 1991; Bradbury et al., 1996). Studies with an acnA-lacZ fusion have shown that expression of the acnA gene is subject to CRP-, Furand SoxRS-mediated activation and ArcA-mediated anaerobic repression (Gruer \& Guest, 1994).

Aconitases are members of a protein family which also contains two analogous types of $\mathrm{Fe}-\mathrm{S}$ enzyme, the isopropylmalate isomerases (IPMIs) and homoaconitases, and two types of vertebrate iron-regulatory protein (IRP), the bifunctional cytoplasmic aconitase/ IRPs (IRP1) and the monofunctional IRPs (IRP2) (Frishman \& Hentze, 1996; Gruer et al., 1997). The IRPs function as iron-dependent translational regulators by binding to specific stem-loop structures (iron-responsive elements, IREs) in relevant mRNA transcripts and in consequence, either blocking translation or in some cases enhancing translation by increasing transcript stability (Hentze \& Kuhn, 1996). Iron-dependent regulation is mediated either by the reversible incorporation of an $\mathrm{Fe}-\mathrm{S}$ cluster in IRP1, which prevents IRE binding and simultaneously generates a cytoplasmic aconitase activity, or in the case of IRP2, by an enzyme-catalysed degradation of the regulator under iron-sufficient conditions.

Sequence comparisons show that E. coli $\mathrm{AcnA}$ is $53 \%$ identical to IRP1 and $27-29 \%$ identical to the mitochondrial aconitases, whereas AcnB is only $15-17 \%$ 
identical to AcnA, IRP and the mitochondrial aconitases. Indeed, E. coli AcnA and the Legionella pneumophila and Bacillus subtilis AcnA proteins, together with the IRPs and plant aconitases, form a discrete IRP-AcnA subfamily whose members are more closely related to each other than to any other members of the aconitase protein family (Gruer $e t$ al., 1997). Likewise, E. coli AcnB and a partially sequenced AcnB from Neisseria gonorrhoeae, form another phylogenetically distinct subfamily that is only remotely related to other members of the family. The overall molecular conformation shared by all members is based on three architecturally distinct arrangements of four structural domains (Bradbury et al., 1996; Gruer et al., 1997). The domain organization predicted for all members of the family except for AcnB and the bacterial IPMIs, is that established by Robbins \& Stout (1989) for porcine heart mitochondrial aconitase, in which three domains $(1,2$ and 3 , tightly packed around the $[\mathrm{Fe}-\mathrm{S}]$ centre, when present) are connected to a C-terminal domain 4 by a long linker in such a way as to form a deep active-site cleft (1-2-3-linker-4). In AcnB it is predicted that domain 4 is located at the $\mathrm{N}$-terminus and connected directly to domain 1 by a short linker (4-linker-1-2-3), and in the bacterial IPMIs, domain 4 exists as an entirely independent subunit $(1-2-3+4)$.

As well as being an important metabolite, citrate is produced commercially from cultures of Aspergillus niger for widespread use as an acidulant in food. Any attempt to engineer bacteria for efficient citrate production will require a greater understanding of bacterial citrate metabolism and the ability to control the conversion of citrate to cis-aconitate and isocitrate via the aconitase reaction. To satisfy this need, and as part of a study aimed at defining the relative roles of $A c n A$ and $A c n B$ in E. coli, $a c n B$ and $a c n A B$ single and double mutants were constructed and their properties compared with those of a previously constructed $a c n A$ mutant.

\section{METHODS}

Bacterial strains, plasmids and phages. The strains of E. coli K-12 and relevant plasmids and phages are listed in Table 1. The $l p d A-a c n B$ region is encoded by a $7.5 \mathrm{~kb} P s t$ fragment in p(iS799 (Fig. 1), a derivative of pUC118 (Bradbury et al., 1996). The source of the $2 \cdot 0 \mathrm{~kb}^{\mathrm{k}} \mathrm{tet}^{\mathrm{R}}$ cassette was pGS969, which was constructed by transferring the cassette as a HindIII fragment from pHP45 $\Omega$-Tc (Fellay et al., 1987) to pMTL24 (Chambers et al., 1988) to pick up flanking $B g l \mathrm{II}$ and $K p n \mathrm{I}$ sites. The acnB-lacZ translational fusion in pGS821 and $\lambda$ G 259 was constructed by PCR-amplification of a $3.69 \mathrm{~kb}$ segment of $\lambda 4 \mathrm{E} 11$ DNA (113 in the mini-set of Kohara et al., 1987) extending across the $a c n B$ gene from the $3^{\prime}$ end of $y a c H$ to the $5^{\prime}$ end of yacL (Fig. 1) and flanked by BamHI-EcoRI and HindIII-PstI sites. The product was purified and cloned as a $3.69 \mathrm{~kb} \mathrm{BamHI}-$ Pst I fragment in pUC119. The sequence of the $916 \mathrm{bp}$ segment containing the $3^{\prime}$ end of $y a c H$, the $y a c H-a c n B$ intergenic region and the first $124 \mathrm{bp}$ of the $a c n B$ coding region, was confirmed and then subcloned as an EcoRI fragment in pNM481 to generate pGS821 (Fig. 1). The fusion was transferred to $\lambda \mathrm{RZ} 5$ (Ostrow et al., 1986) by in vivo recombination in strain RK4353(pGS821) to recover the corresponding fusion phage ( $\lambda \mathrm{G} 259$ ). A $\lambda$ acnB-lacZ monolysogenic derivative of RK4353 designated JRG3253, was selected initially with $\lambda b 80$ del $9 c$ and screened with $\lambda c 190 c 17$ and $\lambda v i r$. An analogous RK4353(2acnA-lacZ) monolysogen designated JRG2744 was constructed previously with $\lambda$ G244 (Gruer \& Guest, 1994). All restriction enzymes were from Northumbria Biologicals.

Media and growth tests. The complex medium was L broth supplemented as required with ampicillin $\left(100 \mu \mathrm{g} \mathrm{ml}^{-1}\right)$, chloramphenicol $\left(25 \mu \mathrm{g} \mathrm{ml}^{-1}\right)$, kanamycin $\left(25 \mu \mathrm{g} \mathrm{ml} \mathrm{g}^{-1}\right)$, nalidixic acid $\left(5 \mu \mathrm{g} \mathrm{ml}^{-1}\right)$, tetracycline $\left(5 \mu \mathrm{g} \mathrm{ml}^{-1}\right)$ and glucose $\langle 0 \cdot 1 \%$ for routine subcultures or $0 \cdot 4 \%$ for anaerobic cultures $)$. The citrate-free minimal medium (Cole \& Guest, 1980) was used with glucose $(20 \mathrm{mM})$, glycerol $(40 \mathrm{mM})$, sodium acetate $(40 \mathrm{mM})$, sodium DL-lactate $(40 \mathrm{mM})$, sodium succinate $(40 \mathrm{mM})$ or sodium pyruvate $(40 \mathrm{mM})$ carbon sources, and supplements of thiamin hydrochloride $\left(5 \mu \mathrm{g} \mathrm{m}^{-1}\right)$, and Lglutamate $(2 \mathrm{mM})$, as required. For anaerobic respiratory growth, sodium fumarate $(40 \mathrm{mM})$ or sodium nitrate $(40 \mathrm{mM})$ were added to glucose minimal medium. For liquid growth tests, cultures $(50 \mathrm{ml})$ were grown at $37^{\circ} \mathrm{C}$, either aerobically in $250 \mathrm{ml}$ conical flasks or anaerobically in stationary bottles. Inocula were prepared from stationary-phase cultures grown in the same medium (with appropriate antibiotics), washed in saline, and added to give a starting $\mathrm{OD}_{650}$ of $0 \cdot 1$ (Unicam SP600 spectrophotometer). For growth tests on solid media, cultures were streaked to single colonies and incubated at $37^{\circ} \mathrm{C}$, growth was recorded at intervals for up to $96 \mathrm{~h}$. Carbon-limited medium containing glucose $(0.0125 \%)$ but no citrate (Davis \& Mingioli, 1950) was used in chemostat cultures $(186 \mathrm{ml})$ inoculated with approximately equal numbers of W3110 (wild-type) and JRG2789 (acnA::kan ${ }^{\mathrm{R}}$ ) bacteria, and fed with fresh medium at 0.2 vols $h^{-1}$. The proportions of each strain were estimated by daily viable counts.

Enzymology. Cell-free extracts were prepared from cultures $(1.5 \mathrm{ml})$ grown as stated, harvested and resuspended in $300 \mu \mathrm{l}$ Tris/citrate buffer $(20 \mathrm{mM}, \mathrm{pH} 8.0)$ at $0{ }^{\circ} \mathrm{C}$, for disruption by two ultrasonic treatments of $10 \mathrm{~s}$ at $10 \mathrm{MHz}$ separated by a $30 \mathrm{~s}$ interval. Cell debris was removed by centrifuging $(10000 \mathrm{~g}, 5 \mathrm{~min})$ and an aliquot $(100 \mu \mathrm{l})$ was taken for protein estimation. DTT $(5 \mathrm{mM}),\left(\mathrm{NH}_{4}\right)_{2} \mathrm{Fe}\left(\mathrm{SO}_{4}\right)_{2}(1 \mathrm{mM})$ and Tris/ $\mathrm{HCl}(50 \mathrm{mM}, \mathrm{pH} 8.0$ ) were added to the major aliquot to stabilize aconitase, which was assayed immediately with $20-100 \mu \mathrm{l}$ samples in reaction mixtures of $1 \mathrm{ml}$ final volume. Aconitase was assayed spectrophotometrically at $240 \mathrm{~nm}$ and $20^{\circ} \mathrm{C}$ by following the conversion of isocitrate to cis-aconitate, using an extinction coefficient of $3.6 \mathrm{mM}^{-1}$ (Kennedy et al., 1983): one unit corresponds to $1 \mu \mathrm{mol}$ cis-aconitate formed $\mathrm{min}^{-1}$. Isocitric acid and cis-aconitate were obtained from Sigma. Published methods were used for assaying other enzymes: citrate synthase (Srere, 1969); isocitrate dehydrogenase (Cribbs \& Englesberg 1964); isocitrate lyase (Dixon \& Kornberg, 1959); citrate lyase and ATP citrate-lyase (Dagley, 1969). Protein concentrations were determined by the Bio-Rad micro-assay procedure. $\beta$-Galactosidase activities were assayed according to the method of Miller (1972) using cultures of $\lambda a c n B-l a c Z$ and $\lambda a c n A-l a c Z$ monolysogens grown aerobically in unsupplemented $L$ broth. Specific activity values (Miller units) are means from at least four independent cultures, the variation between samples being $<15 \%$.

Western blotting. PAGE was performed according to Laemmli $(1970)$ under denaturing conditions $(0 \cdot 1 \%$ SDS in all buffers and $15 \%, \mathrm{w} / \mathrm{v}$, acrylamide) with pre-stained molecular size markers from Sigma (apparent $M_{\mathrm{r}}$ ): $\beta$-galactosidase 
Table 1. E. coli strains, plasmids and phages

\begin{tabular}{|c|c|c|}
\hline $\begin{array}{l}\text { Strain, } \\
\text { plasmid or } \\
\text { phage }\end{array}$ & Relevant characteristics & Source/reference \\
\hline \multicolumn{3}{|l|}{ Strains } \\
\hline W3110 & Prototroph & Laboratory collection \\
\hline JC7623 & recB21 recC22 sbcB15 sbcC201 & Oden et al. (1990) \\
\hline RK4353 & $\Delta(\operatorname{argF}-\operatorname{lac}) \mathrm{U} 169$ & V. Stewart* \\
\hline JRG2744 & גacnA-lacZ ( $\lambda \mathrm{G} 244$ ) monolysogen of RK4353 & Gruer \& Guest (1994) \\
\hline JRG2789 & $a c n A:: k a n^{\mathbf{R}}$-derivative of $\mathrm{W} 3110$ & Gruer \& Guest (1994) \\
\hline JRG3253 & $\lambda a c n B-l a c Z(\lambda \mathrm{G} 259)$ monolysogen of RK4353 & This work \\
\hline JRG3258 & $a c n B:: t^{2} t^{\mathrm{R}}$-derivative of $\mathrm{W} 3110$ & This work \\
\hline JRG3259 & $a c n A:: k a n^{\mathrm{R}} a c n B:: t e t^{\mathrm{R}}$-derivative of $\mathrm{W} 3110$ & This work \\
\hline JRG3359 & $a c n A:: k_{a n}{ }^{\mathrm{R}} a c n B:: t^{2} t^{\mathrm{R}}$ gltA-derivative of W 3110 & This work \\
\hline \multicolumn{3}{|l|}{ Plasmids } \\
\hline pNM481 & lac $Z$ gene fusion vector, $\mathrm{Ap}^{\mathrm{R}}$ & Minton (1984) \\
\hline pG\$799 & $\mathrm{pUC118}, a c n B^{+}, \mathrm{Ap}^{\mathrm{R}}$ & Bradbury et al. (1996) \\
\hline pGS821 & acnB-lacZ-derivative of pNM481, $\mathrm{Ap}^{\mathrm{R}}$ & This work \\
\hline pGS861 & pUC8 acnB:: tet ${ }^{\mathrm{R}}, \mathrm{Ap}^{\mathrm{R}}$ & This work \\
\hline pGS969 & pMTL24-Tet, $A_{p^{R}}$ & This work \\
\hline pGS970 & $\mathrm{pUC8}, a c n B^{+}, \mathrm{Ap}^{\mathrm{R}}$ & This work \\
\hline \multicolumn{3}{|l|}{ Phages } \\
\hline$\lambda \mathrm{G} 244$ & $\lambda a c n A-l a c Z ; A^{\mathrm{R}}$ & Gruer \& Guest (1994) \\
\hline$\lambda \mathrm{G} 259$ & $\lambda a c n B-l a c Z ; A^{\mathrm{R}}$ & This work \\
\hline
\end{tabular}

* Cornell University, Ithaca, NY, USA.

(123000), fructose-6-phosphate kinase (89000), ovalbumin (50000), pyruvate kinase $(67000)$ and triosephosphate isomerase $(34000)$. Samples fractionated by SDS-PAGE were transferred to nitrocellulose with a Bio-R ad Transblot Electrophoretic Transfer Cell. The blots were immuno-stained with either anti-AcnA or anti-AcnB serum as the primary antiserum, biotinylated anti-rabbit antibodies and streptavidinbiotinylated horseradish peroxidase complex (Amersham), according to the method of Prodromou et al. (1991). A Bioprofil image analyser (Vilber-Lourmat) was used for densitometric quantification with calibrations provided by samples of pure $A c n A$ and $A c n B$.

DNA manipulation. DNA was prepared and manipulated by standard procedures (Sambrook et al., 1989). The oligonucleotide primers used in PCR reactions to verify that the $a c n B$ mutant had been successfully constructed were S328 (20221TTGGatCCATA TGCTAGAAGAATACCGTAAGCACGT-

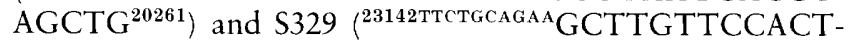
TCATCAAGCAAGGCC $\left.{ }^{23106}\right)$, and S330 (19439TTGGATCCGAATTCAATTGTGGAAAGGCCACCAGTG ${ }^{19462}$ ) was used with S329 in constructing the $a c n B-l a c Z$ fusion; the numbering is for matched bases only (Fujita et al., 1994) and mismatched bases are superscripted. The PCR reactions contained $200 \mathrm{ng}$ template DNA, 100 pmol each primer and $2 \mathrm{UPfu}$ or Taq DNA polymerase (Stratagene).

\section{RESULTS}

\section{Construction of acnB and acnAB mutants}

An $a c n B$ mutant was constructed by replacing the chromosomal $a c n B$ gene of E. coli JC7623 with an in vitro-disrupted $a c n B:: t^{\mathrm{R}}{ }^{\mathrm{R}}$ gene using the method of Oden et al. (1990). The $l p d A-a c n B$ region of pGS799 was first transferred to pUC8 as a $7.5 \mathrm{~kb}$ Pst I fragment to generate pGS970 which lacks the vector $K p n I$ site (Fig. 1). The $1.3 \mathrm{~kb} \mathrm{BglII-KpnI} \mathrm{fragment} \mathrm{of} \mathrm{pGS970} \mathrm{which}$ encodes residues 47-491 of $A c n B$ (i.e. most of domains 4 and 1) was then replaced by a $2 \cdot 0 \mathrm{~kb}$ tet ${ }^{\mathrm{R}}$ cassette from pGS969 (see Methods) to give the pGS861 (Fig. 1). The orientation of the $t e t^{\mathrm{R}}$ gene was defined by mapping the asymmetric Bam HI site relative to flanking sites in pGS861. Replacement of the chromosomal $a c n B^{+}$gene by the $a c n B::$ tet $^{\mathrm{R}}$-derivative was achieved by transforming JC7623 ( $\mathrm{rec} B C \mathrm{~s} b c \mathrm{BC}$ ) with pGS861 and screening Tet $^{\mathrm{R}}$ transformants for potential $a c n B:$ tet $^{\mathrm{R}}$ recombinants which lack the plasmid-encoded ampicillin resistance. The replacement was confirmed by PCR-amplification of the $a c n B$ region of chromosomal DNA from selected recombinants and as controls, JC7623 (parental strain) and two plasmids, pGS861 and pGS799 (Fig. 1). The PCR products were as predicted, $2.9 \mathrm{~kb}$ for JC7623 and pGS799, and $3.6 \mathrm{~kb}$ for the $a c n B:$ : tet $^{\mathrm{R}}$-derivatives of JC7623 and pGS861, reflecting the $0.7 \mathrm{~kb}$ size difference between the tet ${ }^{\mathrm{R}}$ cassette and the $a c n B$ segment that it replaced. The amplified fragments were further characterized by single and double digestion with diagnostic restriction enzymes (BamHI and KpnI) to confirm their origin. The acnB:: tet $^{\mathrm{R}}$ gene of one JC7623-derivative was transferred to E. coli W3110 by P1vir-mediated transduction to produce a representative $a c n B$ mutant, JRG3258 

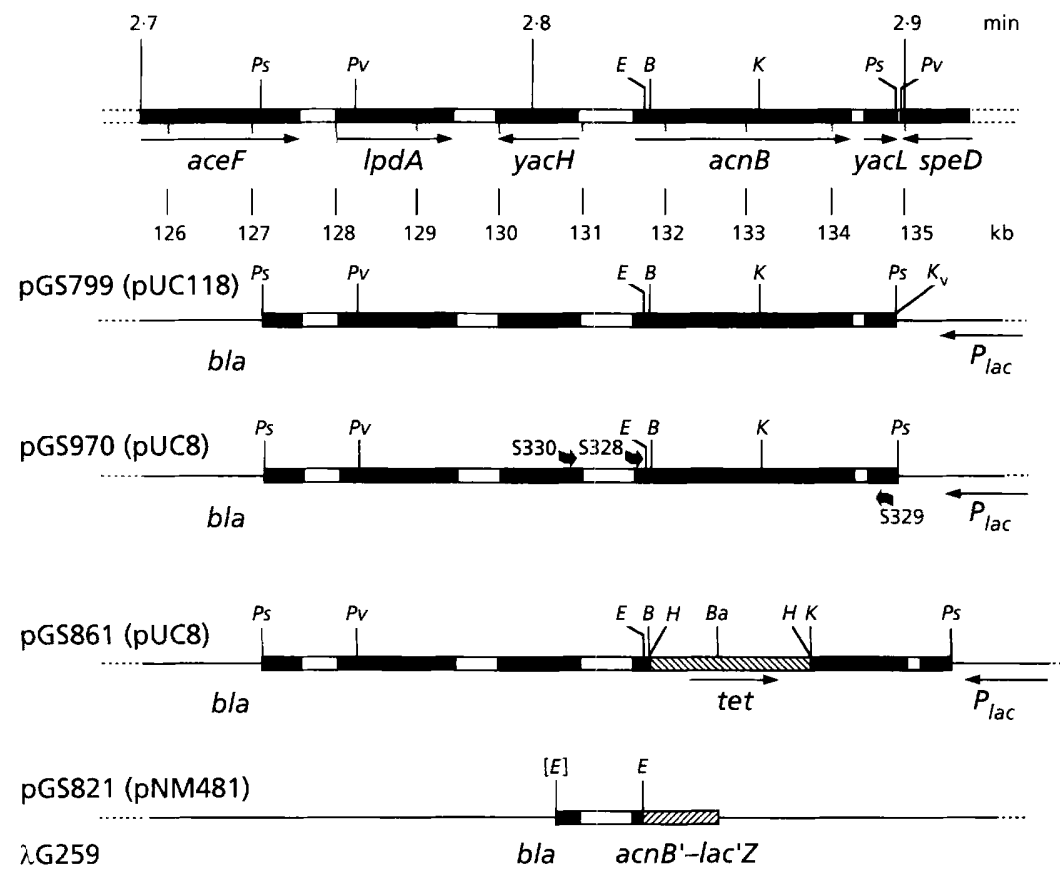

Fig. 1. Strategy for $a c n B$ gene disruption and construction of a hacnB-lacZ fusion phage. Restriction map of the IpdA-acnB region showing segments cloned in specific plasmids (vectors in parentheses), the transcriptional polarities of the genes, and the priming sites for oligonucleotides (shown only on pGS970). Relevant restriction sites are: $B, B g / I I ; B a, B a m H I ; E, E c o R I ; H$, HindIII; K, Kpnl; Ps, Pstl; Pv, Pvull; the subscript $(V)$ refers to flanking vector sites used in subcloning. Artificially created sites are shown in brackets.

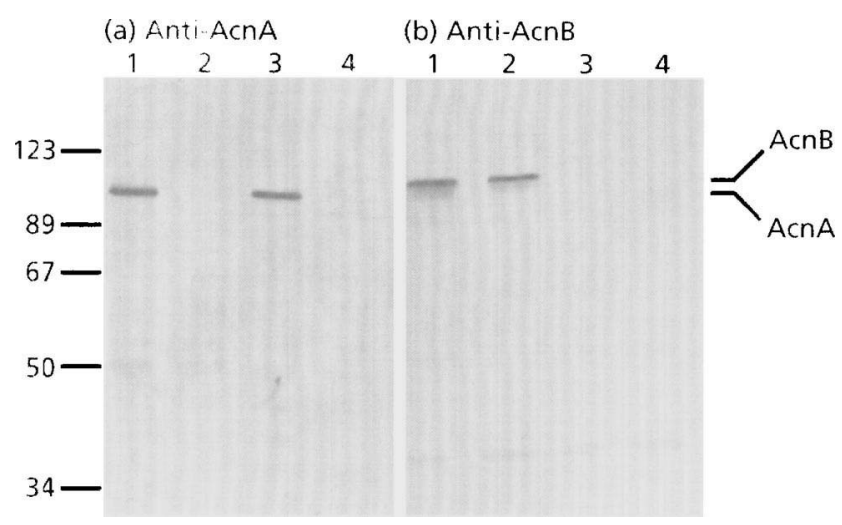

Fig. 2. Western blot analysis of acn mutants. Samples of cellfree extracts of strains grown in L broth plus glucose $(0.1 \%)$ and appropriate antibiotics, were fractionated by SDS-PAGE, blotted and immuno-stained using (a) anti-AcnA serum and (b) anti-AcnB serum. Each lane contained $5 \mu \mathrm{g}$ protein from strain: 1, W3110 $\left(a c n^{+}\right)$; 2, JRG2879 (acnA::kan $\left.a c n B^{+}\right)$; 3, JRG3258

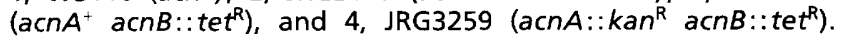
The size calibration was provided by pre-stained markers $\left(10^{-3} \times\right.$ apparent $\left.M_{\mathrm{r}}\right)$.

$\left(a c n B:: t e t^{\mathrm{R}}\right)$. Finally a double mutant, JRG3259 $\left(\operatorname{acn} A:: \operatorname{kan}^{\mathrm{R}} a c n B:: t e t^{\mathrm{R}}\right)$, was constructed by transducing the disrupted $a c n A$ gene of an analogous W3110derivative, JRG2789 (acnA::kan $\left.{ }^{\mathrm{R}}\right)$, into JRG3258 $\left(a c n B:\right.$ tet $^{\mathrm{R}}$ ). Western blot analysis of cell-free extracts of each member of this isogenic set of aconitase mutants confirmed that AcnA is not produced by JRG2789 and JRG3259, nor is AcnB produced by JRG3258 and JRG3259 (Fig. 2).

\section{Nutritional characterization of the acnB and $a c n A B$ mutants}

Citrate synthase $(g l t A)$ and isocitrate dehydrogenase (icd) mutants have an absolute requirement for glutamate for growth on glucose minimal medium, and they cannot use acetate as a sole carbon and energy source either with or without supplementary glutamate (Helling \& Kukora, 1971; Lakshmi \& Helling, 1976). It was expected that aconitase-deficient mutants would exhibit the same phenotype. However, an acnA mutant grew as well as the parental strain in unsupplemented glucose and acetate minimal media, presumably because the $a c n B^{+}$gene complements the $a c n A$ lesion (Gruer \& Guest, 1994). The nutritional phenotypes of the isogenic set of $a c n A, a c n B$ and $a c n A B$ mutants were compared on various types of solid media (Table 2). All of the mutants grew on L agar with and without added glucose under both aerobic and anaerobic conditions, the only detectable difference being that the aerobic growth of the $a c n B$ and $a c n A B$ mutants was slightly impaired. The $a c n A$ mutant was indistinguishable from wild-type on all of the minimal media tested. In contrast, the $a c n B$ mutant and the $a c n A B$ double mutant failed to grow with acetate (Table 2). However, neither of these strains exhibited a clear-cut requirement for glutamate on glucose minimal medium (Table 2). The acnB mutant grew relatively slowly on glucose (and other substrates) and although the rate was enhanced by glutamate, unsupplemented cultures eventually grew to the same extent as the parental strain, indicating that the $\operatorname{acn} \mathrm{A}^{+}$ gene can complement the $a c n B$ lesion, albeit weakly. The $a c n A B$ strain failed to grow on glucose or any of the other substrates tested, and it showed no response to added glutamate except under anaerobic conditions with glucose, where it behaved as a glutamate auxotroph 
Table 2. Nutritional characterization of acn mutants

Cultures were streaked on different solid media spplemented with glutamate (glut) where indicated, and growth was observed for up to $96 \mathrm{~h}$. The relative extents of growth recorded after $40 \mathrm{~h}$, were: $(++)$ good; $(+)$ less good but showing discrete single colonies; $( \pm)$ poor; and $(-)$ no growth. Cultures were grown at $37^{\circ} \mathrm{C}$ under aerobic conditions unless anaerobiosis (ana) is indicated; $(\mathrm{m})$ denotes the presence of a subpopulation of large colonies.

\begin{tabular}{|c|c|c|c|c|}
\hline Medium & $\begin{array}{c}\mathrm{W} 3110 \\
\left(a c n A^{+} a c n B^{+}\right)\end{array}$ & $\begin{array}{c}\text { JRG2789 } \\
\left(\operatorname{acn} A:: \text { kan }^{\mathrm{R}} a c n B^{+}\right)\end{array}$ & $\begin{array}{c}\text { JRG3258 } \\
\left(\operatorname{acn} A^{+} \operatorname{acn} B:: \text { tet }^{\mathrm{R}}\right)\end{array}$ & $\begin{array}{c}\text { JRG3259 } \\
\left(\text { acn } A:: \text { aan }^{\mathrm{R}}\right. \\
\left.\text { acnB:: } \text { tet }^{\mathrm{R}}\right)\end{array}$ \\
\hline L agar & ++ & ++ & ++ & ++ \\
\hline L agar-ana & ++ & ++ & ++ & ++ \\
\hline Glucose (+glut) & $++(++)$ & $++(++)$ & $\pm(+)$ & $-(-/ \mathrm{m})$ \\
\hline Glucose-ana $(+$ glut $)$ & $++(++)$ & $++(++)$ & $\pm(+)$ & $-(+)$ \\
\hline Glycerol (+ glut) & $++(++)$ & $++(++)$ & $\pm(+)$ & $-(-/ \mathrm{m})$ \\
\hline Lactate (+ glut) & $++(++)$ & $++(t+)$ & $\pm(+)$ & $-(-/ \mathrm{m})$ \\
\hline Pyruvate (+glut) & $+(+)$ & $+(+)$ & $\pm(+)$ & $-(-)$ \\
\hline Succinate $(+$ glut $)$ & $+(+)$ & $+(+)$ & $\pm(+)$ & $-(-/ \mathrm{m})$ \\
\hline Acetate (+glut) & $+(+)$ & $+(+)$ & $-(-)$ & $-(-)$ \\
\hline
\end{tabular}

(Table 2). Another striking feature of the acn $A B$ double mutant was the appearance of a substantial population of fast-growing 'revertant' or secondary mutant colonies during aerobic incubation on glutamatesupplemented glucose minimal medium (Table 2). These 'revertants' eventually amounted to approximately $50 \%$ of the bacteria plated. They also appeared on other glutamate-supplemented media but were never detected with unsupplemented media. The 'revertants' were not detected during anaerobic growth, or with the $a c n B$ single mutant, possibly because the mutants grew sufficiently well under the respective conditions. Further studies with the 'revertants' are described below.

Quantitative growth tests in $\mathrm{L}$ broth showed that the effects of the $a c n A:: k a n^{\mathrm{R}}$ mutation are negligible compared with the increasing debility wrought by the $a c n B:: t e t^{\mathrm{R}}$ mutation alone and in combination with the $a c n A:: k a n^{\mathrm{R}}$ mutation (Fig. 3a). The impairments were lessened in the presence of glucose (Fig. 3b) and abolished during anaerobic growth in L broth with or without glucose (data not shown). Likewise in glucose minimal medium, the $a c n A:: k a n^{\mathrm{R}}$ lesion had no apparent effect (Fig. 3c) nor did the corresponding strain, JRG2789 $\left(a c n A:: \operatorname{kan}^{\mathrm{R}}\right)$, show any detectable disadvantage when competed with W3110 $\left(a c n A^{+}\right)$over 40 generations in glucose-limited continuous culture (data not shown). In contrast, JRG3258 (acnB:: tet $^{\mathrm{R}}$ ) and JGR3259 (acnA:: kan ${ }^{\mathrm{R}}$ acnB::tet $\left.{ }^{\mathrm{R}}\right)$ were severely impaired in glucose minimal medium (Fig. 3c). The $a c n B$ mutant eventually achieved a high cell density after prolonged incubation and, as observed with solid media, growth was slightly enhanced by adding glutamate (Fig. 3c). The double mutant showed a very weak response to glutamate before being over-grown by the fast-growing 'revertants'. However, under anaerobic conditions, added glutamate increased the growth rates of the $a c n B$ and $a c n A B$ mutants and allowed them to achieve the same yields as the $a c n A$ and $a c n^{+}$strains (Fig. 3d).

\section{Enzymological characterization of acn mutants}

The aconitase specific activities reproducibly observed for W3110, JRG2789 (acnA::kan $\left.{ }^{\mathrm{R}}\right)$ and JRG3258 $\left(a c n B:: t e t^{\mathrm{R}}\right)$ indicated that $A \subset n B$ is synthesized earlier in the growth cycle than $A c n A$ and that $A c n B$ is the major aconitase (Fig. 4). It also appeared that the absence of one enzyme may increase the synthesis of the other, because the sum of the activities of the individual mutants exceeded that of the parent at most times in the growth cycle. A small but significant aconitase activity ranging from 2 to $5 \%$ of wild-type was always detected in cultures of JRG3259 (acnA::kan ${ }^{\mathrm{R}}$ acnB::tet ${ }^{\mathrm{R}}$ ) suggesting that there may be a third aconitase, AcnC (Table 3). The specific activities of several related enzymes were unaffected by the $a c n$ mutations except for a $60 \%$ lowering of isocitrate lyase in the double mutant. No ATP citrate-lyase activity $[<0.001 \mathrm{U}(\mathrm{mg}$ protein $\left.)^{-1}\right]$ was detected in any of the strains after aerobic or anaerobic growth.

\section{Regulation of AcnB synthesis}

To study the synthesis of $A c n B$ in the presence of $A c n A$, a $\lambda a c n B-l a c Z$ fusion phage ( $\lambda \mathrm{G} 259)$ containing the $a c n B$ promoter and part of the coding region fused 'in-phase' to a $\beta$-galactosidase gene, was constructed (Fig. 1). The $\beta$-galactosidase activities expressed from the corresponding $\lambda a c n B-l a c Z$ monolysogen of RK4353, JRG3253 (acn $A^{+} B^{+} \Delta$ lacZ), grown in different media, are listed in Table 4 . In the rich medium, $a c n B$ gene expression was repressed by glucose and anaerobiosis like a typical citric acid cycle gene. The profile of activities observed after growth in other media, particularly the high value with acetate, was likewise consistent with $A c n B$ performing a major role in the citric acid and glyoxylate cycles.

Studies with comparable $\lambda a c n A-l a c Z$ and $\lambda a c n B-l a c Z$ lysogens (JRG2744 and JRG3253, respectively) con- 

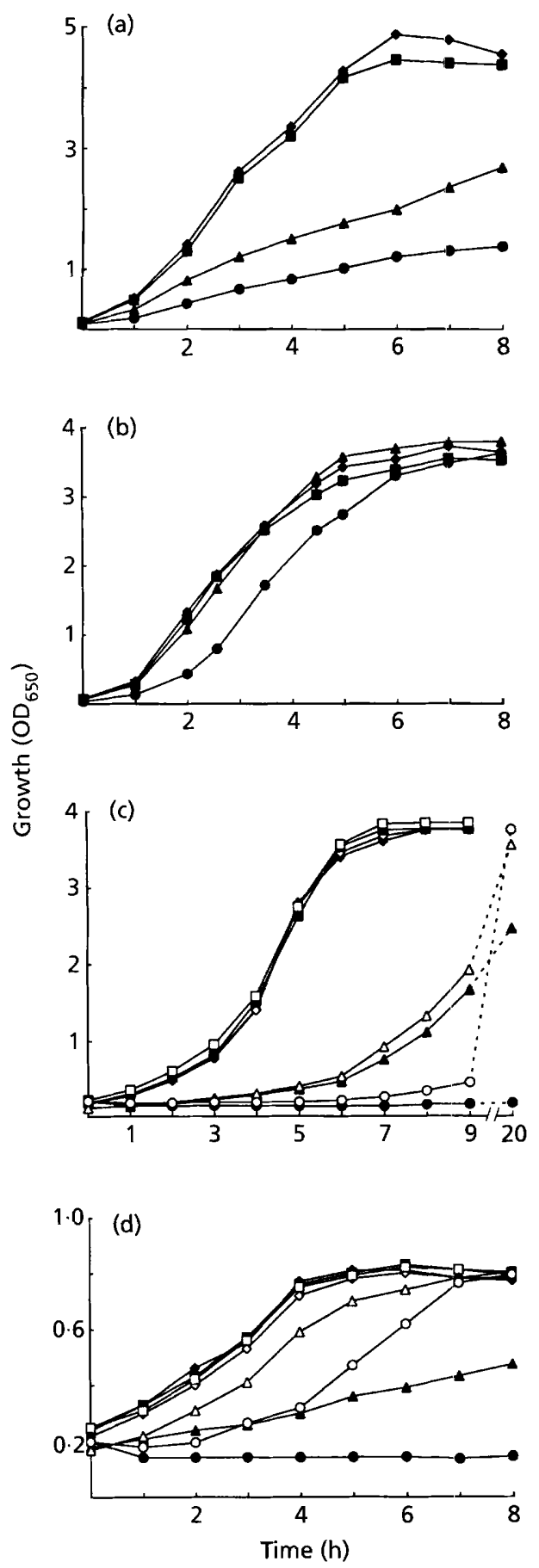

Fig. 3. Growth of acn mutants and the parental strain under different conditions. Typical results are shown for cultures of W3110 (acnA $a c n B^{+}$; ), JRG2789 (acnA::kan $\left.{ }^{\mathrm{R}} a c n B^{+} ; \square\right)$, JRG3258 (acnA $A^{+}$acnB::tet $\left.{ }^{R} ; \Delta\right)$ and JRG3259 (acnA::kan ${ }^{R}$ acnB::tet ${ }^{R}$; ) grown at $37^{\circ} \mathrm{C}$ in: (a) aerobic $\mathrm{L}$ broth; (b) aerobic $L$ broth containing $0.4 \%$ glucose; $(c)$ aerobic glucose minimal medium, unsupplemented (closed symbols) or supplemented with glutamate (open symbols); (d) anaerobic glucose minimal medium, unsupplemented (closed symbols) or supplemented with glutamate (open symbols).

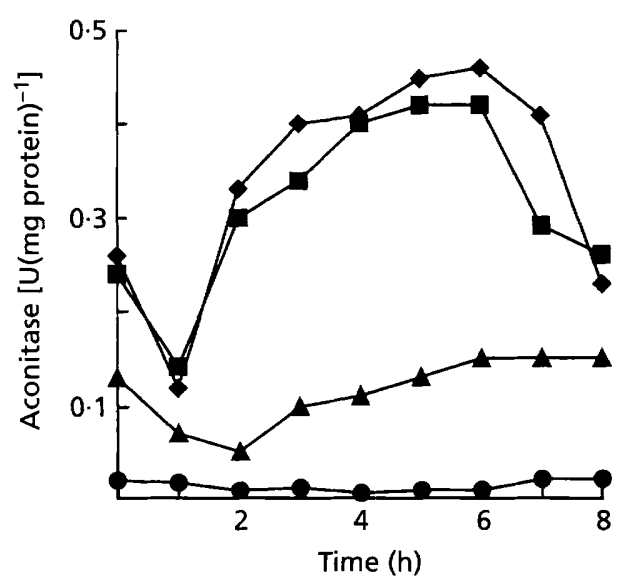

Fig. 4. Aconitase activities of acn mutants. The aerobic L-broth cultures shown in Fig. 3(a) were sampled periodically for measuring aconitase specific activity: $\bullet, W 3110\left(a c n A^{+} a c n B^{+}\right)$; 口. JRG2789 (acnA::kan $\left.a c n B^{+}\right) ; \quad \boldsymbol{A}, J R G 3258 \quad\left(a c n A^{+}\right.$ acnB:: tet $\left.t^{R}\right)$; and O, JRG3259 (acnA::kan $\left.a c n B:: t^{R} t^{R}\right)$.

firmed that $a c n B$ expression precedes $a c n A$ expression (Fig. 5a). The $a c n B$ gene was activated early in the exponential phase and the decline that accompanied entry into the stationary phase almost coincided with the activation of the $a c n A$ gene. The maximum activity generated by the $a c n B-l a c Z$ fusion was approximately 11 -fold greater than that produced by the acnA-lac $Z$ fusion (Fig. 5a). Assuming that these activities truly reflect those of the aconitases, the results strongly suggest that $A c n B$ is the major aconitase. Quantitative Western blot analyses using specific polyclonal antisera with the parental strain (W3110) further confirmed that AcnB synthesis precedes AcnA (Fig. 5b). However, the maximum amount of $A c n B$ protein was no more than threefold greater than that of AcnA. The discrepancy between the ratios of Acn-LacZ fusion activities and the Acn protein contents could be due to differences in protein stability and turnover. Alternatively, the expression of one or both of the acn-lacZ fusions could be differentially affected by the presence of two copies of the respective promoter in the monolysogens $\mathrm{PaCn}^{+}$ $\left.\lambda \mathrm{PaCn}^{+}\right)$. The effects of one aconitase on the synthesis of the other were investigated immunochemically by comparing the amounts of each protein in equivalent samples of mutant and wild-type bacteria (Fig. 6). This showed that the AcnA protein concentration in the acnB::tet ${ }^{\mathbf{R}}$ mutant was very similar to that in the parental strain (Fig. 6b) but the AcnB concentration in the $a c n A:: k_{a n}{ }^{\mathrm{R}}$ mutant was approximately twofold lower than wildtype (Fig. 6c). The reason for this difference is not known but it could mean that AcnA has a positive regulatory effect on $A c n B$ synthesis.

\section{Properties of ' revertants' or secondary mutants}

Lakshmi \& Helling (1976) observed that icd mutants are rapidly over-grown by double mutants (icd glt $A$ ) which lack citrate synthase. Both lesions confer glutamate 
Table 3. Enzymology of aconitase mutants

Enzyme specific activities [ $\mathrm{U}(\mathrm{mg} \text { protein })^{-1}$ ] were determined with representative extracts of stationary-phase L-broth cultures grown at $37^{\circ} \mathrm{C}$ under aerobic conditions unless stated otherwise: values are means from three determinations with different amounts of extract. No ATP citrate-lyase was detected in any of the extracts.

\begin{tabular}{|c|c|c|c|c|c|}
\hline Enzyme & $\begin{array}{c}\text { W3110 } \\
\left(a c n A^{+} a c n B^{+}\right)\end{array}$ & $\begin{array}{c}\text { JRG2789 } \\
\left(\operatorname{acn} A:: \operatorname{kan}^{\mathrm{R}} \operatorname{acnB}^{+}\right)\end{array}$ & $\begin{array}{c}\text { JRG3258 } \\
\left(a c n A^{+} a c n B:: t e t^{\mathrm{R}}\right)\end{array}$ & $\begin{array}{c}\text { JRG3259 } \\
\left(a c n A:: k_{a n}{ }^{\mathrm{R}}\right. \\
\left.\operatorname{acn} B:: \operatorname{tet}^{\mathrm{R}}\right)\end{array}$ & $\begin{array}{c}\text { JRG3359 } \\
\left(a c n A:: k_{a n}^{\mathrm{R}}\right. \\
\left.\operatorname{acnB}:: \operatorname{tet}^{\mathrm{R}} \text { glt } A\right)\end{array}$ \\
\hline Aconitase & $0 \cdot 46$ & $0 \cdot 41$ & $0 \cdot 15$ & $0 \cdot 02$ & $0 \cdot 017$ \\
\hline Citrate synthase & 0.56 & 0.52 & 0.47 & 0.65 & $<0.001$ \\
\hline Isocitrate dehydrogenase & $1 \cdot 06$ & 0.95 & $0 \cdot 97$ & $0 \cdot 95$ & 0.57 \\
\hline Citrate lyase & $0 \cdot 001$ & $0 \cdot 001$ & 0.001 & $<0 \cdot 001$ & $<0.001$ \\
\hline Citrate lyase (anaerobic) & 0.002 & 0.002 & $0 \cdot 002$ & $0 \cdot 001$ & 0.001 \\
\hline Isocitrate lyase & $0 \cdot 50$ & $0 \cdot 48$ & 0.55 & $0 \cdot 21$ & $0 \cdot 40$ \\
\hline
\end{tabular}

Table 4. Effects of growth conditions on acnB-lacZ expression

$\beta$-Galactosidase specific activities (Miller units) expressed by cultures of a $2 a c n B-$ lacZ monolysogen, RK4353( $\lambda$ G259), in the late-exponential/early-stationary transitory phase. Means are given for four independent cultures; the deviation was less than $15 \%$.

\begin{tabular}{|lc|}
\hline Medium & $\boldsymbol{\beta}$-Galactosidase \\
\hline L broth & 400 \\
L broth + glucose & 105 \\
L broth (anaerobic) & 55 \\
L broth + glucose (anaerobic) & 35 \\
Glucose minimal & 106 \\
Glycerol minimal & 235 \\
Succinate minimal & 237 \\
Acetate minimal & 376 \\
Glucose + fumarate (anaerobic) & 80 \\
Glucose + nitrate (anaerobic) & 30 \\
\hline
\end{tabular}

auxotrophy but the inability to produce citrate clearly improved growth in the presence of glutamate. The icd mutants were originally isolated by virtue of their resistance to low concentrations of nalidixic acid $(10 \mu \mathrm{g}$ $\mathrm{ml}^{-1}$, Helling \& Kukora, 1971) and it was later observed that the icd gltA secondary mutants re-acquire the parental sensitivity to concentrations above $2 \mu \mathrm{g} \mathrm{ml}^{-1}$ (Lakshmi \& Helling, 1976). The intracellular citrate concentration was increased several hundredfold in $i c d$ mutants and lowered in the icd glt A mutants, so it was suggested that the accumulation of citrate (or a derivative) renders the $i c d$ mutant resistant to nalidixic acid. Consistent with these observations, all of four independent $a c n A B$ ' revertants' selected on glucose plus glutamate agar lacked citrate synthase activity as well as aconitase. The enzymological phenotype of a typical acnAB glt $A$ mutant (JRG3359) is summarized in Table 3. It shows that citrate synthase is no longer detectable whereas the activities of related enzymes are essentially
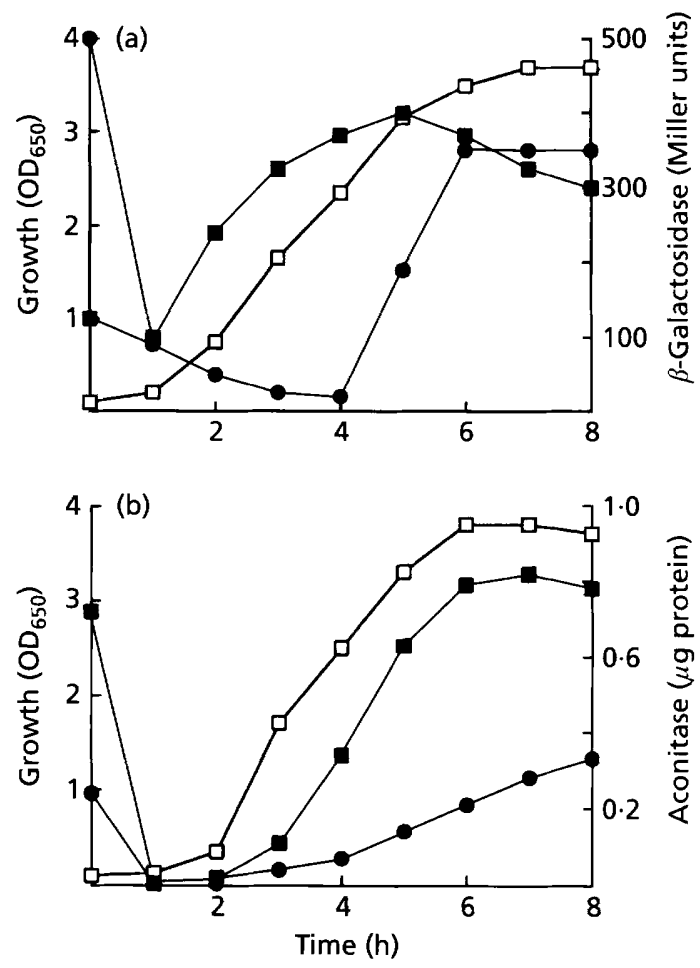

Fig. 5. Comparison of $A C n A$ and $A C n B$ synthesis in the growth cycle. (a) Growth ( $\square$ ) and $\beta$-galactosidase activities expressed from single-copy acnA-lacZ (multiplied by $10 ; 0$ ) and $a c n B-l a c Z$ ( $\boldsymbol{\Xi})$ fusion prophages in aerobic L-broth cultures of RK4353 monolysogens (means from four independent cultures). (b) Immunochemical comparison of the amounts of aconitase protein produced by W3110 during aerobic growth in L broth: $\square$, growth $\left(O D_{650}\right) ; \boldsymbol{Q}, A c n A ; \square, A c n B$. Samples equivalent to $15 \mu$ l of a bacterial suspension at $O D_{650}=1.0$ were withdrawn and analysed by quantitative Western blotting using either anti-AcnA or anti-AcnB serum.

unchanged, except for a $45 \%$ lowering of isocitrate dehydrogenase. The triple mutants grew almost as well as W3110 on glutamate-supplemented glucose medium, and they used all of the other test substrates except 

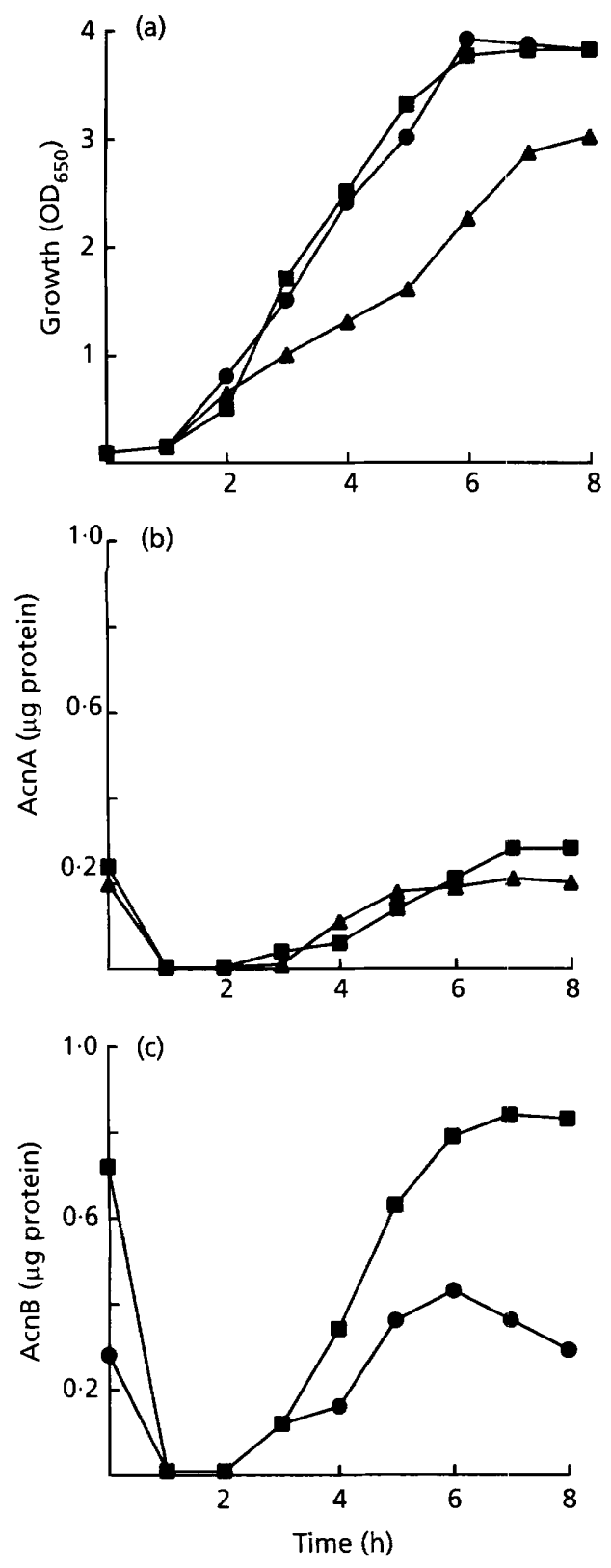

Fig. 6. Effects of acn mutations on AcnA or AcnB synthesis. Growth (a) and amounts of AcnA (b) or AcnE (c) proteins in samples of aerobic L-broth cultures (equivalent to $15 \mu \mathrm{l}$ of a bacterial suspension at $O D_{650}=1.0$ ) were estimated by quantitative densitometry of Western blots with: $\mathbf{0}$, W3110 $\left(a c n A^{+} a c n B^{+}\right) ; 0, J R G 2789\left(a c n A:: k a n^{R} a c n B^{+}\right) ; A^{\prime}, J R G 3258$ $\left(a c n A^{+} a c n B:: \operatorname{tet}^{R}\right)$.

acetate if supplemented with glutamate. However, although the $a c n B$ and $a c n A B$ mutants were resistant to higher concentrations of nalidixic acid than the parental or acnA strains $(5 \mu \mathrm{g} \mathrm{ml})^{-1}$ relative to $\left.2 \mu \mathrm{g} \mathrm{ml} l^{-1}\right)$, the $a c n A B$ glt $A$ 'revertants' differed from their icd gltA counterparts in retaining the $\mathrm{Nal}^{\mathrm{R}}$ phenotype. The reason for the discrepancy is not clear. It could mean that the $\mathrm{Nal}^{\mathrm{R}}$ phenotype is not solely dependent on citrate accumulation. Fourteen $\operatorname{acn} A B$ glt $A$-derivatives were tested, so it is unlikely that all have acquired an independent mutation conferring resistance to nalidixic acid. It seems more likely that the effects of the acn and icd mutations are not entirely analogous, or that there are underlying differences between parental strains. The window for estimating the $\mathrm{Nal}^{\mathrm{S}} / \mathrm{Nal}^{\mathrm{R}}$ phenotype is narrow and it may be significant that an independent glt $A$ mutant was almost as resistant to nalidixic acid as the $a c n A B$ mutant studied here (Lakshmi \& Helling, 1976).

If growth of the $a c n A B$ mutant is inhibited by the accumulation of high intracellular concentrations of citrate, it was envisaged that some of the 'revertants' might have increased their ability to tolerate citrate, possibly by inducing new routes for citrate dissimilation or excretion. No such secondary mutants were detected, but their existence cannot be excluded because the number of 'revertants' tested was relatively small. It would also appear that the seemingly toxic effects of citrate accumulation are not bactericidal, because the viability of the $a c n A B$ double mutant remained approximately constant in aerobic glucose minimal medium without or with glutamate for $30 \mathrm{~h}$ or until reversion occurred, respectively.

The absence of growth of the $\operatorname{acn} A B$ double mutant (JRG3259) in glucose minimal medium offered the possibility of selecting prototrophic revertants. It was envisaged that such revertants might arise if the putative AcnC activity could be induced to levels that would complement the lack of AcnA and AcnB. Revertants of this type were induced by adding a few crystals of NTG to the centres of plates of glucose minimal medium enriched with nutrient broth $(0 \cdot 2 \%, \mathrm{v} / \mathrm{v})$ and spread with the double mutant $\left(10^{8}\right.$ bacteria). Prototrophic revertant colonies appeared at a frequency of $4 \times 10^{-8}$, but only after anaerobic incubation. They grew to different extents under anaerobic conditions, and although growth was improved by adding glutamate it was never as good as that obtained with W3110. These prototrophs were unable to grow aerobically on unsupplemented glucose minimal medium and their response to added glutamate ranged from negligible to weak. Enzymological studies with seven of these conditional anaerobic prototrophic revertants showed that their aconitase activities were two- to fourfold higher than in the double mutant. If this elevation is due to the third aconitase, such strains might serve as usefully enriched sources of AcnC.

\section{DISCUSSION}

Inactivation of the $a c n A$ and $a c n B$ genes has revealed some interesting and hitherto unsuspected features of the citric acid cycle of $E$. coli. Most notable was the retention of a low residual aconitase activity in the $a c n A B$ double mutant. It is not known whether the activity is due to a third aconitase, AcnC, or to an unrelated hydratase-dehydratase having a broad substrate specificity that includes citrate. The absence of a clear-cut glutamate auxotrophy, except under anaerobic 
conditions, was also unexpected. It explains why aconitase mutants were not previously isolated as glutamate-requiring mutants along with those lacking citrate synthase $(g l t A)$. This is not simply due to the fact that $a c n A$ and $a c n B$ mutants are capable of growing on unsupplemented glucose minimal medium, but also because the double mutant is unable to grow aerobically on glutamate-supplemented medium (unless citrate synthase is inactivated by yet another mutation). The latter observation further explains the failure of previous attempts to isolate $a c n A B$ double mutants by starting with the acnA:: $\mathrm{kan}^{\mathrm{R}}$ single mutant (Gruer, 1993). Random transposon mutagenesis, followed by either direct nutritional screening or initial selections for $\mathrm{Nal}^{\mathrm{R}}$. derivatives, was used to detect aerobic glutamate auxotrophs. However, although icd acnA::kan ${ }^{\mathrm{R}}$ double mutants with $\mathrm{Glt}^{-} \mathrm{Nal}^{\mathrm{R}}$ phenotypes were recovered (some with enhanced aconitase activities), no aconitasedeficient auxotrophs were detected.

The failure of $a c n A B$ double mutants to respond to glutamate (i.e. absence of a Glt ${ }^{-}$phenotype) seems to be due to the toxic accumulation of citrate or a derivative of citrate, because growth occurs when citrate synthesis is blocked. This situation seems to represent a more extreme case of that observed previously with icd mutants (Lakshmi \& Helling, 1976). The icd mutants are glutamate auxotrophs which generate icd glt A doubles spontaneously, whereas the aconitase-deficient strain $(a c n A B)$ cannot grow unless citrate synthase is inactivated. The partially deficient $a c n B$ mutant occupies an intermediate position in growing (albeit slowly) without glutamate, and in responding only weakly to this supplement. The problems posed by the multiplicity of aconitases and citrate toxicity will have to be surmounted if a successful process for citrate production is to be developed with E. coli.

The response to glutamate was much clearer under anaerobic conditions and no acn $A B$ glt $A$ 'revertants' were detected. This could mean that under anaerobic conditions, citrate is exported at a rate that prevents its accumulation in inhibitory concentrations, or that $\mathrm{AcnC}$ might be induced sufficiently to divert citrate to other products, though not sufficiently to satisfy the need for glutamate. The two- to fourfold increase in $\mathrm{AcnC}$ activity in anaerobic $\mathrm{Glt}^{+}$revertants is consistent with the latter explanation, especially as it allows some aerobic growth in the presence of glutamate (i.e. it offsets the toxic effects of citrate) without abolishing the need for glutamate.

The enzymological, immunological and acn-lacZ fusion studies consistently indicated that $A c n B$ is the major citric acid cycle and glyoxylate cycle enzyme. This contradicts an earlier conclusion that $A c n B$ represents only $10-30 \%$ of total aconitase activity (Gruer \& Guest, 1994). The discrepancy, and the fact that AcnA was the first aconitase to be isolated from E. coli, is almost certainly due to the greater stability of AcnA relative to $\mathrm{AcnB}$ (unpublished observations). In contrast, AcnA seems to perform a specific role in redox-stress and stationary phase. It is not known whether AcnA performs a regulatory role like its close relative IRP1, or whether AcnB serves as a highly labile redox sensor. It is conceivable that one or both apo-proteins might exhibit site-specific nucleic acid binding and thus mediate an iron- or redox-dependent transcriptional or translational switch. The observation that AcnB synthesis is lower in the acn $A$ mutant suggested that AcnA positively regulates $A c n B$ synthesis, but this has not been substantiated in recent studies which showed that the $\operatorname{acn} A:: k_{a n}{ }^{\mathrm{R}}$ mutation has no significant effect on the expression of the $\lambda a c n B-l a c Z$ fusion (unpublished observations). Evidence is now being sought at the in vivo and in vitro levels for potential regulatory roles for $A c n A$ and $A c n B$. The nature of $A c n C$ and the control of $a c n B$ promoter activity are likewise being investigated.

\section{ACKNOWLEDGEMENTS}

We are very grateful to J. Green, M. A. Quail and R. B. Helling for advice and helpful discussions. The work was supported by grants from the Wellcome Trust and by a studentship from the Biotechnology Directorate of the Biotechnology and Biological Sciences Research Council (A. J.B.).

\section{REFERENCES}

Bradbury, A. J., Gruer, M. J., Rudd, K. E. \& Guest, J. R. (1996). The second aconitase (AcnB) of Escherichia coli. Microbiology 142, 389-400.

Chambers, S. P., Prior, S. E., Barstow, D. A. \& Minton, N. P. (1988). The pMTL nic ${ }^{-}$cloning vectors. 1 . Improved pUC polylinker regions to facilitate the use of sonicated DNA for nucleotide sequencing. Gene 68, 139-149.

Cole, S. T. \& Guest, J. R. (1980). Genetic and physical characterization of lambda transducing phages ( $\lambda \mathrm{frdA}$ ) containing the fumarate reductase gene of Escherichia coli K12. Mol Gen Genet 178, 409-418.

Cribbs, R. \& Englesberg, E. (1964). L-Arabinose negative mutants of the L-ribulokinase structural gene affecting the levels of Larabinose isomerase in Escherichia coli. Genetics 49, 95-108.

Dagley, S. (1969). Citrate lyase. Methods Enzymol 13, 160-163. Davis, B. D. \& Mingioli, E. S. (1950). Mutants of Escherichia coli requiring methionine or vitamin $\mathrm{B}_{12} . J$ Bacteriol 60, 17-28.

Dixon, G. H. \& Kornberg, H. L. (1959). Assay methods for key enzymes of the glyoxylate cycle. Biochem J 72, 3P.

Fellay, R., Frey, J. \& Krisch, H. (1987). Interposon mutagenesis of soil and water bacteria. A family of DNA fragments designed for in vitro insertional mutagenesis of Gram-negative bacteria. Gene 52, 147-154.

Frishman, D. \& Hentze, M. W. (1996). Conservation of aconitase residues revealed by multiple sequence analysis. Implications for structure/function relationships. Eur J Biochem 239, 197-200.

Fujita, N., Mori, H., Yura, T. \& Ishihama, A. (1994). Systematic sequencing of the Escherichia coli genome: analysis of the 2.4 4.1 min (110917-193643 bp) region. Nucleic Acids Res 22, $1637-1639$.

Gruer, M. J. (1993). Molecular genetic characterisation of Escherichia coli aconitase. PhD thesis, University of Sheffield.

Gruer, M. J. \& Guest, J. R. (1994). Two genetically-distinct and 
differentially-regulated aconitases (AcnA and $\mathrm{AcnB}$ ) in Escherichia coli. Microbiology 140, 2531-2541.

Gruer, M. J., Artymiuk, P. J. \& Guest, J. R. (1997). The aconitase family: three structural variations on a common theme. Trends Biochem Sci 22, 3-6.

Helling, R. B. \& Kukora, J. S. (1971). Nalidixic acid-resistant mutants of Escherichia coli deficient in isocitrate dehydrogenase. J Bacteriol 105, 1224-1226.

Hentze, M. W. \& Kuhn, L. C. (1996). Molecular control of vertebrate iron metabolism: mRNA-based regulatory controls operated by iron, nitric oxide, and oxidative stress. Proc Natl Acad Sci USA 93, 8175-8182.

Kennedy, M. C., Emptage, M. H., Dreyer, J.-L. \& Bienert, H. (1983). The role of iron in the activation-inactivation of aconitase. J Biol Chem 258, 11098-11105.

Kohara, Y., Akiyama, K. \& Isono, K. (1987). The physical map of the whole E. coli chromosome: application of a new strategy for rapid analysis and sorting of a large genomic library. Cell 50, 495-508.

Laemmli, U. K. (1970). Cleavage of structural proteins during the assembly of the head of bacteriophage T4. Nature 277, 680-685.

Lakshmi, T. M. \& Helling, R. B. (1976). Selection for citrate synthase deficiency in icd mutants of Escherichia coli. J Bacteriol $127,76-83$.
Miller, J. H. (1972). Experiments in Molecular Genetics. Cold Spring Harbor, NY: Cold Spring Harbor Laboratory.

Minton, N. (1984). Improved plasmid vectors for the isolation of translational lac gene fusions. Gene 31, 269-273.

Oden, K. L., DeVeaux, L. C., Vibat, C. R. T., Cronan, J. E. \& Gennis, R. B. (1990). Genomic replacement in Escherichia coli K-12 using covalently closed circular plasmid DNA. Gene 96, 29-36.

Ostrow, K. S., Silhavy, T. J. \& Garrett, S. (1986). Cis-acting sites required for osmoregulation of $o m p F$ expression in Escherichia coli K-12. J Bacteriol 168, 1165-1171.

Prodromou, C., Haynes, M. J. \& Guest, J. R. (1991). The aconitase of Escherichia coli: purification of the enzyme and molecular cloning and map location of the gene (acn). J Gen Microbiol 137, 2505-2515.

Robbins, A. H. \& Stout, C. D. (1989). The structure of aconitase. Proteins 5, 289-312.

Sambrook, J., Fritsch, E. F. \& Maniatis, T. (1989). Molecular Cloning: a Laboratory Manual, 2nd edn. Cold Spring Harbor, NY: Cold Spring Harbor Laboratory.

Srere, P. A. (1969). Citrate synthase. Methods Enzymol 13, 3-11.

Received 4 December 1996; revised 24 January 1997; accepted 28 January 1997. 